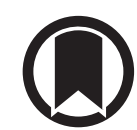

CrossMark

\title{
Prognostic impact of copeptin in pulmonary embolism: a multicentre validation study
}

\author{
Kristian Hellenkamp¹, Piotr Pruszczyk², David Jiménez (103), Anna Wyzgat², \\ Deisy Barrios ${ }^{3}$, Michał Ciurzyński ${ }^{2}$, Raquel Morillo ${ }^{3}$, Lukas Hobohm ${ }^{4,5}$, \\ Karsten Keller ${ }^{4}$, Katarzyna Kurnicka2, Maciej Kostrubiec ${ }^{2}$, Rolf Wachter ${ }^{6,7}$, \\ Gerd Hasenfuß ${ }^{1,6}$, Stavros Konstantinides ${ }^{4}$ and Mareike Lankeit ${ }^{1,4,8,9}$
}

Affiliations: ${ }^{1}$ Clinic of Cardiology and Pneumology, Heart Centre, University Medical Center Goettingen, Goettingen, Germany. ${ }^{2}$ Dept of Internal Medicine and Cardiology, Medical University of Warsaw, Warsaw, Poland. ${ }^{3}$ Respiratory Dept, Ramón y Cajal Hospital, Alcalá de Henares University, IRYCIS, Madrid, Spain. ${ }^{4}$ Centre for Thrombosis and Haemostasis (CTH), University Medical Centre Mainz, Johannes GutenbergUniversity Mainz, Mainz, Germany. ${ }^{5}$ Centre for Cardiology, Cardiology I, University Medical Centre Mainz, Johannes Gutenberg-University Mainz, Mainz, Germany. ${ }^{6}$ German Cardiovascular Research Centre (DZHK), Goettingen, Germany. ${ }^{7}$ Clinic and Policlinic for Cardiology, University Hospital Leipzig, Leipzig, Germany. ${ }^{8}$ Dept of Internal Medicine and Cardiology, Campus Virchow Klinikum (CVK), Charité - University Medicine, Berlin, Germany. ${ }^{9}$ German Cardiovascular Research Centre (DZHK), Berlin, Germany.

Correspondence: Mareike Lankeit, Dept of Internal Medicine and Cardiology, Campus Virchow Klinikum (CVK), Charité - University Medicine Berlin, Augustenburger Platz 1, 13353 Berlin, Germany.

E-mail: mareike.lankeitacharite.de

@ERSpublications

Copeptin improves risk stratification of normotensive patients with pulmonary embolism http://ow.ly/ 8E3P30jtvym

Cite this article as: Hellenkamp K, Pruszczyk P, Jiménez D, et al. Prognostic impact of copeptin in pulmonary embolism: a multicentre validation study. Eur Respir J 2018; 51: 1702037 [https://doi.org/ 10.1183/13993003.02037-2017].

ABSTRACT To externally validate the prognostic impact of copeptin, either alone or integrated in risk stratification models, in pulmonary embolism (PE), we performed a post hoc analysis of 843 normotensive PE patients prospectively included in three European cohorts.

Within the first 30 days, 21 patients $(2.5 \%, 95 \%$ CI 1.5-3.8) had an adverse outcome and $12(1.4 \%, 95 \%$ CI 0.7-2.5) died due to PE. Patients with copeptin $\geqslant 24 \mathrm{pmol} \cdot \mathrm{L}^{-1}$ had a 6.3 -fold increased risk for an adverse outcome (95\% CI 2.6-15.5, p<0.001) and a 7.6-fold increased risk for PE-related death (95\% CI 2.3-25.6, $\mathrm{p}=0.001$ ). Risk classification according to the 2014 European Society of Cardiology (ESC) guideline algorithm identified 248 intermediate-high-risk patients (29.4\%) with 5.6\% (95\% CI 3.1-9.3) at risk of adverse outcomes. A stepwise biomarker-based risk assessment strategy (based on high-sensitivity troponin T, $\mathrm{N}$-terminal pro-brain natriuretic peptide and copeptin) identified 123 intermediate-high-risk patients (14.6\%) with $8.9 \%$ (95\% CI 4.5-15.4) at risk of adverse outcomes. The identification of patients at higher risk was even better when copeptin was measured on top of the 2014 ESC algorithm in intermediate-high-risk patients (adverse outcome OR 11.1, 95\% CI 4.6-27.1, p<0.001; and PE-related death OR 13.5, 95\% CI 4.2-43.6, $\mathrm{p}<0.001$; highest risk group versus all other risk groups). This identified 85 patients $(10.1 \%)$ with $12.9 \%(95 \%$ CI 6.6-22.0) at risk of adverse outcomes and 8.2\% (95\% CI 3.4-16.2) at risk of PE-related deaths.

Copeptin improves risk stratification of normotensive PE patients, especially when identifying patients with an increased risk of an adverse outcome.

This article has supplementary material available from erj.ersjournals.com

Received: Oct 052017 | Accepted after revision: Feb 282018

Copyright OERS 2018 


\section{Introduction}

The 2014 guideline of the European Society of Cardiology (ESC) recommends risk stratification of patients with acute pulmonary embolism (PE), because this can influence treatment decisions [1]. While haemodynamically unstable PE patients are easily identified as being at high risk, risk stratification in initially normotensive $\mathrm{PE}$ is more challenging. A multimodal approach based on a clinical risk prediction score (e.g. the simplified Pulmonary Embolism Severity Index (sPESI) [2]), testing of cardiac biomarkers and imaging of the right ventricle is proposed to classify normotensive patients into low, intermediate-low or intermediate-high-risk groups [1]. However, this ESC algorithm is complex and may be insufficient to identify higher risk patients who potentially may profit from a more aggressive treatment strategy. Thus, in the past years, several attempts have been made to optimise risk stratification in normotensive PE [3-6].

Right ventricular (RV) dysfunction due to the sudden increase of RV afterload is considered a critical determinant of PE severity, because it can lead to a decreased left ventricular preload and, subsequently, to shock. While cardiac biomarkers such as high-sensitivity troponin $\mathrm{T}$ (hsTnT) and N-terminal pro-brain natriuretic peptide (NT-proBNP) reflect myocardial injury or stretch [7,8], vasopressin (AVP), and its more stable surrogate marker copeptin, might reflect a promising new pathophysiological axis of PE severity because AVP is released on stress and hypotension and may therefore indicate impaired haemodynamics due to RV failure $[9,10]$. In a prospective single-centre derivation study including 268 normotensive PE patients [9], we showed that elevated copeptin levels using an optimal cut-off value of $24 \mathrm{pmol} \cdot \mathrm{L}^{-1}$ were associated with a 5.4-fold increased risk of an adverse 30-day outcome. The odds ratio for an adverse outcome was even higher if copeptin was combined with the cardiac biomarkers hsTnT and NT-proBNP in a stepwise biomarker-based risk assessment strategy.

The aim of the present European multicentre study was to validate the prognostic impact of copeptin in normotensive PE. In particular, we aimed to validate a stepwise biomarker-based risk assessment strategy combining cardiac biomarkers and copeptin and to investigate the prognostic impact of copeptin for the identification of normotensive PE patients at higher risk of an adverse 30-day outcome.

\section{Methods}

\section{Patient population and study design}

At each cooperating site (provided in the supplementary material), consecutive patients aged $\geqslant 18$ years with objectively confirmed acute symptomatic PE were prospectively enrolled in local ongoing non-interventional cohort studies. For the present post hoc analysis, only normotensive (systolic blood pressure $\geqslant 90 \mathrm{mmHg}$ on admission) patients were included. Patients with missing blood samples, invalid biomarker measurements and German patients previously included in the derivation study [9] were excluded from analysis. All sites followed the same study protocol (as described in [7]), allowing the patient cohorts to be pooled. Information on the definitions used in the present study are provided in the supplementary material.

Patients were stratified to risk classes according to the sPESI [2], 2014 ESC algorithm [1], biomarker-based risk assessment strategy [9] and Bova score [3]; missing values were considered to be normal.

The primary outcome was an adverse 30-day outcome, defined as PE-related death or at least one of the following complications: need for 1) catecholamine administration, 2) mechanical ventilation or 3) cardiopulmonary resuscitation. The secondary outcomes were defined as PE-related or all-cause death within 30 days. All outcomes, including causes of death, were adjudicated by local independent adjudication committees. Death was determined to be PE-related if it was either confirmed by autopsy or followed a clinically severe episode of acute PE in the absence of an alternative diagnosis.

The study was conducted in accordance with the amended Declaration of Helsinki; the study protocol was approved by the local independent ethics committees of each participating site and all patients gave written informed consent. Treatment decisions were made by the physicians caring for the patients and were not influenced by the study protocol. Study results were not communicated to the treating physicians and thus not used to guide the patients' management or to monitor the effects of treatment at any time.

\section{Laboratory biomarker testing}

Venous blood samples were collected on admission and immediately stored at $-80^{\circ} \mathrm{C}$. Plasma levels of copeptin, hsTnT and NT-proBNP were measured post hoc as described previously [9] and in the supplementary material. Elevated biomarker concentrations were prospectively defined as hsTnT $\geqslant 14 \mathrm{pg} \cdot \mathrm{mL}^{-1}$ [7], NT-proBNP $\geqslant 600 \mathrm{pg} \cdot \mathrm{mL}^{-1}$ [8] and copeptin $\geqslant 24 \mathrm{pmol} \cdot \mathrm{L}^{-1}$ [9]. 


\begin{abstract}
Statistical analysis
Analyses were performed using SPSS (version 23.0; SPSS Inc., Chicago, IL, USA). Categorical variables are presented as n (\%); study outcomes are given as absolute risk (\%) with corresponding 95\% confidence intervals calculated using Clopper-Pearson "exact" intervals. Comparison of categorical variables was performed with Fisher's exact test. The Kolmogorov-Smirnov test revealed that continuous variables did not follow a normal distribution. They are therefore presented as median (interquartile range (IQR)). The Mann-Whitney U-test was used for comparison of continuous variables. Receiver operating characteristics (ROC) analysis was performed and the area under the curve (AUC) determined to test the performance of copeptin with regard to the prediction of an adverse outcome and PE-related death. Youden's index quantification was used to identify the optimal cohort-specific cut-off values. Univariable logistic regression analysis was used to calculate the odds ratio and the corresponding $95 \%$ confidence intervals of variables with regard to the prediction of an adverse outcome and of PE-related death. For this purpose, risk models (2014 ESC algorithm, Bova score, biomarker-based strategy, modified 2014 ESC algorithm and 2014 ESC algorithm with subsequent copeptin measurement in intermediate-high-risk patients) were dichotomised by testing patients classified in the highest risk group versus patients stratified in any other risk group (combination of low-risk and intermediate-low-risk class). Additionally, parameters univariably associated with an adverse outcome were separately tested in combination with copeptin $\geqslant 24 \mathrm{pmol} \cdot \mathrm{L}^{-1}$ in multivariable logistic regression models. Sensitivity, specificity, negative predictive values, positive predictive values, negative and positive likelihood ratios and the corresponding $95 \%$ confidence intervals were calculated. All statistical tests were two-sided and used a significance level of 0.05 .
\end{abstract}

TABLE 1 Baseline characteristics, medical history and initial presentation of the study patients

\begin{tabular}{|c|c|c|c|c|}
\hline & All study patients & Copeptin $<24 \mathrm{pmol} \cdot \mathrm{L}^{-1}$ & Copeptin $\geqslant 24 \mathrm{pmol} \cdot \mathrm{L}^{-1}$ & p-value \\
\hline Subjects $\mathbf{N}$ & 843 & 662 & 181 & \\
\hline Age years median (IQR) & $70.0(53.0-79.0), n=841$ & $67.0(50.0-77.0), n=661$ & $77.0(67.0-82.5), n=180$ & $<0.001$ \\
\hline BMI $\mathrm{kg} \cdot \mathrm{m}^{-2}$ median (IQR) & $26.8(24.0-30.0), n=620$ & $26.7(23.7-30.1), n=483$ & $27.0(24.4-30.0), n=137$ & 0.357 \\
\hline \multicolumn{5}{|l|}{ Comorbidities } \\
\hline Active cancer & $126(15.0), n=842$ & 95 (14.4), $n=662$ & 31 (17.2), n=180 & 0.347 \\
\hline Renal insufficiency & $244(29.2), n=836$ & $159(24.1), n=659$ & 85 (48.0), $n=177$ & $<0.001$ \\
\hline \multicolumn{5}{|l|}{ Symptoms } \\
\hline Signs/symptoms of DVT & $221(26.2)$ & $189(28.5)$ & 32 (17.7) & 0.003 \\
\hline Chest pain & 365 (43.3), n=842 & $300(45.4), n=661$ & 65 (35.9), $n=181$ & 0.028 \\
\hline Dyspnoea & 645 (76.6), $n=842$ & $501(75.8), n=661$ & $144(79.6), n=181$ & 0.322 \\
\hline Syncope & 133 (15.8), $n=842$ & 89 (13.5), $n=661$ & 44 (24.3), $n=181$ & 0.001 \\
\hline RV dysfunction (on TTE) & 195 (25.5), $n=766$ & $142(23.2), n=611$ & 53 (34.2), $n=155$ & 0.007 \\
\hline RV dysfunction (on TTE or MDCT) & $267(32.9), n=811$ & $192(30.1), n=638$ & 75 (43.4), n=173 & 0.001 \\
\hline \multicolumn{5}{|l|}{ Laboratory biomarkers } \\
\hline hsTnT pg $\cdot \mathrm{mL}^{-1}$ median (IQR) & $19.3(7.9-44.7)$ & $14.7(6.6-35.2)$ & $40.7(24.3-84.2)$ & $<0.001$ \\
\hline $\mathrm{hsTnT} \geqslant 14 \mathrm{pg} \cdot \mathrm{mL}^{-1}$ & $504(59.8)$ & $344(52.0)$ & $160(88.4)$ & $<0.001$ \\
\hline NT-proBNP $\mathrm{pg} \cdot \mathrm{mL}^{-1}$ median (IQR) & $449(115-2257)$ & $290(95-1342)$ & $1787(431-7246)$ & $<0.001$ \\
\hline NT-proBNP $\geqslant 600 \mathrm{pg} \cdot \mathrm{mL}^{-1}$ & $376(44.6)$ & $250(37.8)$ & $126(69.6)$ & $<0.001$ \\
\hline \multicolumn{5}{|l|}{ Risk classes } \\
\hline sPESI $\geqslant 1$ point & $495(58.7)$ & $348(52.6)$ & $147(81.2)$ & $<0.001$ \\
\hline \multicolumn{5}{|l|}{2014 ESC algorithm" } \\
\hline Low risk & 135 (16.0) & $126(19.0)$ & $9(5.0)$ & $<0.001$ \\
\hline Intermediate-low risk & $460(54.6)$ & $373(56.3)$ & $87(48.1)$ & 0.053 \\
\hline Intermediate-high risk & $248(29.4)$ & $163(24.6)$ & $85(47.0)$ & $<0.001$ \\
\hline
\end{tabular}




\section{Results}

\section{Study cohort and patient outcomes}

Between August 2007 and May 2016, 883 patients were included in the study; of those, 30 were excluded because of missing blood samples and 10 because of invalid biomarker measurements. Thus, 843 normotensive PE patients (496 from Spain, 252 from Poland and 95 from Germany) were included in the present analysis. The medical history and baseline characteristics of the study patients are provided in table 1. An echocardiographic examination was performed in 766 patients (90.9\%); of those, 195 (25.5\% of patients with transthoracic echocardiography (TTE), $23.1 \%$ of all patients) were diagnosed with RV dysfunction. During the first 30 days, the primary outcome (adverse 30-day outcome) occurred in 21 patients $(2.5 \%, 95 \%$ CI $1.5-3.8)$, all-cause death in 36 patients $(4.3 \%, 95 \%$ CI $3.0-5.9)$ and PE-related death in 12 patients $(1.4 \%, 95 \%$ CI $0.7-2.5)$.

\section{Prognostic impact of copeptin in normotensive PE}

Copeptin plasma concentrations ranged from 1.0 to $380.1 \mathrm{pmol} \cdot \mathrm{L}^{-1}$ (median $9.6 \mathrm{pmol} \cdot \mathrm{L}^{-1}$, IQR $5.2-21.1$ ). Differences of patients with copeptin levels above the predefined cut-off value of $24 \mathrm{pmol} \cdot \mathrm{L}^{-1}$ compared to patients with copeptin levels $<24 \mathrm{pmol} \cdot \mathrm{L}^{-1}$ are shown in table 1 . The median copeptin concentrations were higher in patients with an adverse outcome or PE-related death than in patients with a favourable outcome (32.7 pmol. $\mathrm{L}^{-1}$, IQR $8.8-52.3$ versus $9.5 \mathrm{pmol} \cdot \mathrm{L}^{-1}$, IQR $5.2-20.4, \mathrm{p}<0.001$; and $31.5 \mathrm{pmol} \cdot \mathrm{L}^{-1}$, IQR 9.3-64.8 versus $9.5 \mathrm{pmol} \cdot \mathrm{L}^{-1}$, IQR 5.2-20.7, $\left.\mathrm{p}<0.001\right)$ and more patients with an adverse outcome or PE-related death had copeptin $\geqslant 24 \mathrm{pmol} \cdot \mathrm{L}^{-1}(61.9 \%, 95 \%$ CI $38.4-81.9$ versus $20.4 \%$, 95\% CI $17.7-23.4$, $\mathrm{p}<0.001$; and 66.7\%, 95\% CI 34.9-90.1 versus 20.8\%, 95\% CI 18.1-23.7, $\mathrm{p}=0.001$ ) compared to patients with a favourable clinical course. Patients with copeptin $\geqslant 24 \mathrm{pmol} \cdot \mathrm{L}^{-1}$ more often had an adverse outcome (7.2\%, 95\% CI $3.9-12.0$ versus $1.2 \%, 95 \%$ CI $0.5-2.4 ; \mathrm{p}<0.001)$ or died of PE $(4.4 \%, 95 \%$ CI $1.9-8.5$ versus $0.6 \%, 95 \%$ CI $0.2-1.5 ; \mathrm{p}=0.001)$ compared to patients with copeptin $<24 \mathrm{pmol} \cdot \mathrm{L}^{-1}$. The prognostic performance of copeptin and other risk assessment tools with regard to an adverse outcome are shown in table 2. Using ROC analysis, the AUC for copeptin was 0.70 (95\% CI $0.57-0.83, \mathrm{p}=0.002$ ) for predicting an adverse outcome and 0.71 (95\% CI $0.55-0.88, \mathrm{p}=0.011)$ for predicting PE-related death. In comparison, the AUC for predicting an adverse outcome for hsTnT was 0.69 (95\% CI 0.58-0.79, p=0.004) and for NT-proBNP was 0.72 (95\% CI $0.58-0.85, \mathrm{p}=0.001$ ) (figure 1 ). In univariate logistic regression analysis, copeptin $\geqslant 24 \mathrm{pmol} \cdot \mathrm{L}^{-1}$ was associated with a more than six-fold increased risk of an adverse outcome and an almost eight-fold increased risk for PE-related death (table 3). Further univariable predictors of primary or secondary study outcomes are shown in table 3 . The prognostic value of copeptin $\geqslant 24 \mathrm{pmol} \cdot \mathrm{L}^{-1}$ to predict an adverse outcome or PE-related death remained independent if tested with each variable associated with these outcomes separately in multivariable logistic models (data not shown). Similar results were obtained if patient cohorts of the derivation [9] and the present studies were pooled (supplementary material).

Copeptin helps to identify normotensive PE patients with an elevated risk for adverse outcomes Using a simple stepwise biomarker-based strategy, 504 patients (59.8\%) were classified as low risk with a rate of an adverse outcome of $1.0 \%$ (95\% CI 0.3-2.3) (figure 2a). Specifically, one patient ( $0.2 \%$ of all low-risk patients) died of $\mathrm{PE}$, three patients required administration of catecholamines and two patients required mechanical ventilation. Using the 2014 ESC algorithm, only 135 patients (16.0\%) were classified as low risk; none of them had an adverse outcome (figure 2b). At the other end of the risk spectrum, the 2014 ESC algorithm classified 248 patients (29.4\%) as being at intermediate-high risk; of those, 14 (5.6\%, 95\% CI 3.1-9.3) had an adverse outcome and nine (3.6\%, 95\% CI 1.7-6.8) died of PE. In comparison,

TABLE 2 Prognostic performance of dichotomised laboratory biomarkers, RV dysfunction according to imaging modalities and sPESI with regard to an adverse 30 -day outcome

\begin{tabular}{|c|c|c|c|c|c|c|}
\hline & Sensitivity & Specificity & PPV & NPV & $\mathrm{LR}+\#$ & LR-\# \\
\hline $\mathrm{hsTnT} \geqslant 14 \mathrm{pg} \cdot \mathrm{mL}^{-1}$ & 90 (71-97) & $41(38-44)$ & $4(2-6)$ & 99 (98-100) & $1.5(1.3-1.8)$ & $0.2(0.1-0.9)$ \\
\hline $\mathrm{NT}-$ proBNP $\geqslant 600 \mathrm{pg} \cdot \mathrm{mL}^{-1}$ & $81(60-92)$ & $56(53-60)$ & $5(3-7)$ & $99(98-100)$ & $1.9(1.5-2.3)$ & $0.3(0.1-0.8)$ \\
\hline sPESI $\geqslant 1$ point & $90(71-97)$ & $42(39-45)$ & $4(2-6)$ & 99 (98-100) & $1.6(1.3-1.8)$ & $0.2(0.1-0.8)$ \\
\hline RV dysfunction (on TTE) & $44(23-67)$ & 75 (72-78) & $4(2-7)$ & $98(97-99)$ & $1.7(1.0-3.1)$ & $0.8(0.5-1.2)$ \\
\hline RV dysfunction (on TTE or MDCT) & $48(28-67)$ & $67(64-71)$ & $4(2-7)$ & $98(96-99)$ & $1.5(0.9-2.3)$ & $0.8(0.5-1.2)$ \\
\hline
\end{tabular}

Data are presented as $\%(95 \% \mathrm{Cl})$, unless otherwise stated. PPV: positive predictive value; NPV: negative predictive value; LR+/-: positive/ negative likelihood ratio; hsTnT: high-sensitivity troponin T; NT-proBNP: N-terminal pro-brain natriuretic peptide; sPESI: simplified Pulmonary Embolism Severity Index; RV: right ventricular; TTE: transthoracic echocardiography; MDCT: multidetector computed tomography. ${ }^{\#}$ : data presented as ratio $(95 \% \mathrm{Cl})$. 
patients classified as intermediate-high risk using the biomarker-based strategy had a higher rate of adverse outcomes of $8.9 \%$ (95\% CI 4.5-15.4) and PE-related deaths of 5.7\% (95\% CI 2.3-11.4).

To combine the successful performance of the 2014 ESC algorithm in identifying low-risk patients and the more effective identification of normotensive PE patients at higher risk using copeptin, we tested whether copeptin measurement on top of the 2014 ESC algorithm could further improve risk stratification of patients classified as intermediate-high risk. As shown in figure $2 \mathrm{c}$, when using copeptin $\geqslant 24 \mathrm{pmol} \cdot \mathrm{L}^{-1}$ to further stratify patients in the intermediate-high-risk group, 85 patients $(10.1 \%)$ were identified as being at higher risk with a rate of adverse outcome of $12.9 \%$ (95\% CI 6.6-22.0) and of PE-related death of $8.2 \%$ (95\% CI 3.4-16.2) while 163 patients were reclassified as intermediate-low risk. Given that not all hospitals will have TTE always available for the assessment of RV dys(function), and evidence of RV dysfunction on TTE was not associated with an increased risk of an adverse outcome in the present study (OR 2.3, 95\% CI 0.9-6.3, $\mathrm{p}=0.099$ ), we tested whether replacing information from imaging modalities with copeptin measurements in the 2014 ESC algorithm could provide comparable prognostic information. These results are shown in supplementary figure $1 \mathrm{~s}$.

In summary, the odds ratios determined using logistic regression analysis for risk of adverse outcome or PE-related death, respectively, for patients classified to the highest risk groups based on 1) the biomarker-based strategy (figure 2a) were 7.0 (95\% CI 2.9-16.8, p<0.001) and 8.6 (95\% CI 2.7-27.6, $\mathrm{p}<0.001$ ), 2) the 2014 ESC algorithm (figure $2 \mathrm{~b}$ ) were 5.0 (95\% CI 2.0-12.6, p=0.001) and 7.4 (95\% CI 2.0-27.7, $\mathrm{p}=0.003$ ), 3) the modified 2014 ESC algorithm (using copeptin instead of imaging in the 2014 ESC algorithm, supplementary figure 1s) were 7.4 (95\% CI 3.1-18.0, p<0.001) and 7.6 (95\% CI 2.4-24.2, $\mathrm{p}=0.001)$ and 4) the 2014 ESC algorithm followed by measurement of copeptin (figure 2c) were $11.1(95 \%$ CI 4.6-27.1, p<0.001) and 13.5 (95\% CI 4.2-43.6, p<0.001) (table 3). Figure 3 gives an overview of the percentage of patients with an adverse outcome and PE-related death in each risk class using different risk assessment strategies.

\section{Discussion}

We performed a post hoc analysis of a large pooled European multicentre cohort to validate the prognostic impact of copeptin in 843 normotensive patients with acute PE. The main study findings can be summarised as follows: 1) copeptin using a predefined cut-off value of $24 \mathrm{pmol} \cdot \mathrm{L}^{-1}$ had a good prognostic performance and was associated with a 6.3-fold increased risk for an adverse outcome and a 7.6-fold increased risk for PE-related death; 2) established risk assessment strategies such as the 2014 ESC algorithm safely identify PE patients at low risk while a stepwise biomarker-based risk assessment strategy combining hsTnT, NT-proBNP and copeptin appears especially useful to identify normotensive PE patients with a higher risk of adverse outcomes; and 3) risk stratification of normotensive PE can be optimised if copeptin is measured on top of the 2014 ESC algorithm.

FIGURE 1 Receiver operating characteristics analysis for biomarkers with regard to an adverse 30-day outcome. Area under the curve for copeptin (pmol. $\mathrm{L}^{-1}$ ), high-sensitivity troponin $\mathrm{T}$ (hsTnT) ( $\mathrm{pg} \cdot \mathrm{mL}^{-1}$ ) and $\mathrm{N}$-terminal pro-brain natriuretic peptide (NT-proBNP) $\left(\mathrm{pg} \cdot \mathrm{mL}^{-1}\right.$ ) with regard to an adverse 30-day outcome.

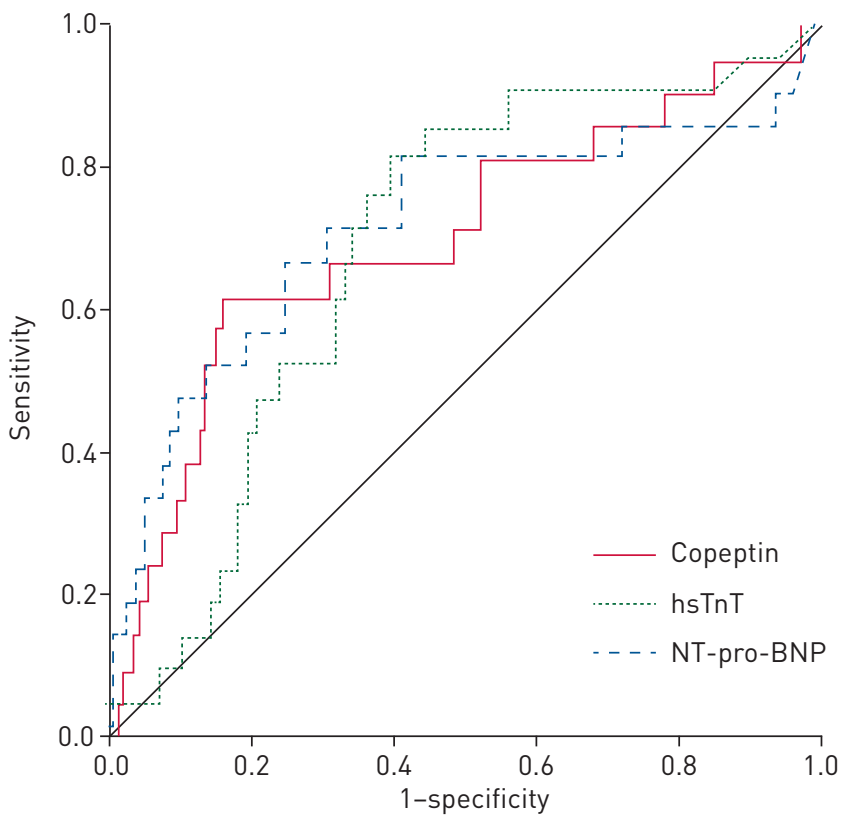


TABLE 3 Predictors of adverse outcomes

\begin{tabular}{|c|c|c|c|c|c|c|}
\hline & \multicolumn{2}{|c|}{ Adverse 30-day outcome } & \multicolumn{2}{|c|}{ PE-related death } & \multicolumn{2}{|c|}{ All-cause death } \\
\hline Subjects $(\%, 95 \%$ Cl) & $21(2.5,1.5-3.8)$ & & $12(1.4,0.7-2.5)$ & & $36(4.3,3.0-5.9)$ & \\
\hline \multicolumn{7}{|l|}{ Comorbidities } \\
\hline Chronic heart failure & $5.57(2.08-14.93)$ & 0.001 & $2.61(0.56-12.21)$ & 0.222 & $2.73(1.09-6.84)$ & 0.032 \\
\hline Chronic pulmonary disease & $3.56(1.34-9.42)$ & 0.011 & $2.88(0.77-10.86)$ & 0.117 & $3.54(1.65-7.61)$ & 0.001 \\
\hline Chest pain & $0.32(0.11-0.96)$ & 0.043 & $1.09(0.33-3.60)$ & 0.887 & $0.42(0.20-0.91)$ & 0.027 \\
\hline \multicolumn{7}{|l|}{ Laboratory biomarkers } \\
\hline $\mathrm{hs} T \mathrm{TT} \geqslant 14 \mathrm{pg} \cdot \mathrm{mL}^{-1}$ & $6.60(1.53-28.53)$ & 0.011 & & & $4.38(1.69-11.38)$ & 0.002 \\
\hline $\mathrm{NT}-$ proBNP $\geqslant 600 \mathrm{pg} \cdot \mathrm{mL}^{-1}$ & $5.48(1.83-16.43)$ & 0.002 & $14.04(1.81-109.28)$ & 0.012 & $4.62(2.08-10.25)$ & $<0.001$ \\
\hline Copeptin $\geqslant 24 \mathrm{pmol} \cdot \mathrm{L}^{-1}$ & $6.33(2.58-15.51)$ & $<0.001$ & $7.61(2.26-25.56)$ & 0.001 & $4.45(2.26-8.75)$ & $<0.001$ \\
\hline \multicolumn{7}{|l|}{ Risk classes } \\
\hline $\begin{array}{l}2014 \text { ESC algorithm plus } \\
\text { copeptin: intermediate-high risk }{ }^{\#}\end{array}$ & $11.12(4.57-27.05)$ & $<0.001$ & 13.52 (4.19-43.59) & $<0.001$ & $7.52(3.71-15.24)$ & $<0.001$ \\
\hline $\begin{array}{l}\text { Modified } 2014 \text { ESC algorithm: } \\
\text { intermediate-high risk }\end{array}$ & $7.44(3.07-18.01)$ & $<0.001$ & 7.55 (2.36-24.15) & 0.001 & $5.78(2.92-11.43)$ & $<0.001$ \\
\hline
\end{tabular}

Data are presented as OR $(95 \% \mathrm{CI})$, unless otherwise stated. Bold font indicates statistical significance. PE: pulmonary embolism; hsTnT: high-sensitivity troponin T; NT-proBNP: N-terminal pro-brain natriuretic peptide; sPESI: simplified Pulmonary Embolism Severity Index; ESC: European Society of Cardiology. "\#: intermediate-high risk tested versus intermediate-low and low risk.

Validation of the prognostic impact of copeptin in normotensive PE

Copeptin as a surrogate marker for AVP was identified as a helpful novel biomarker for rapid rule-out of myocardial infarction [11, 12]. Additionally, it has been shown to predict prognosis in acute coronary syndrome [13], chronic heart failure [14, 15], pulmonary hypertension [16] and, recently, acute PE [9, 17, 18]. In heart failure $[14,15]$, copeptin was superior to natriuretic peptides with regard to the prediction of death. In patients with acute myocardial infarction, KHAN et al. [13] demonstrated that copeptin was a predictor of death, especially if combined with NT-proBNP. The authors of the latter article underline that "a multimarker strategy [...] using independent biomarkers has benefits in that it integrates the different pathways involved, in the hope that complementary information can be gained" [13]. In acute PE, the sudden increase of RV afterload may lead to a decrease in left ventricular preload and subsequently to low cardiac output and shock. Given that AVP, together with copeptin, is released upon stress and hypotension $[9,10]$, this may reflect a novel pathophysiological axis of PE severity by indicating the systemic response to impaired haemodynamics due to RV failure.

Although the rate of an adverse 30-day outcome was lower in the present study compared to the derivation studies (2.5\% in this study compared to $5.6 \%$ in [9] and $9.3 \%$ in [17]), we were able to validate the prognostic impact of elevated copeptin plasma concentrations. Of note, this direct comparison might be biased given the different endpoint definitions. However, in agreement with the derivation studies, patients with an adverse outcome had higher copeptin plasma concentrations on admission and elevated copeptin levels were associated with a $>6$-fold increased risk for an adverse outcome (derivation study OR 5.4, 95\% CI 1.7-17.6 [9]). In the present study, copeptin was identified as a predictor of PE-related death, supporting the concept that copeptin provides information on the haemodynamic impairment due to acute RV failure (which might require more aggressive treatment regimens) [18]. Importantly, the prognostic value of copeptin remained independent if tested separately with other variables associated with an adverse outcome or PE-related death in multivariable logistic models.

When using a predefined cut-off value of $24 \mathrm{pmol} \cdot \mathrm{L}^{-1}$, only $21.5 \%$ of the study patients had elevated copeptin, whereas hsTnT and NT-proBNP were elevated in as many as $59.8 \%$ and $44.6 \%$ of patients, respectively. Thus, and as shown in table 2, hsTnT and NT-proBNP were associated with better sensitivity than copeptin. These biomarkers may therefore be useful to identify low-risk patients, whereas copeptin 
a) Biomarker-based strategy

Normotensive patients with acute $\mathrm{PE} n=843$

Adverse outcome $\mathrm{n}=21(2.5 \%, 95 \% \mathrm{Cl} 1.5-3.8)$

PE-related death $\mathrm{n}=12(1.4 \%, 95 \% \mathrm{Cl} 0.7-2.5)$

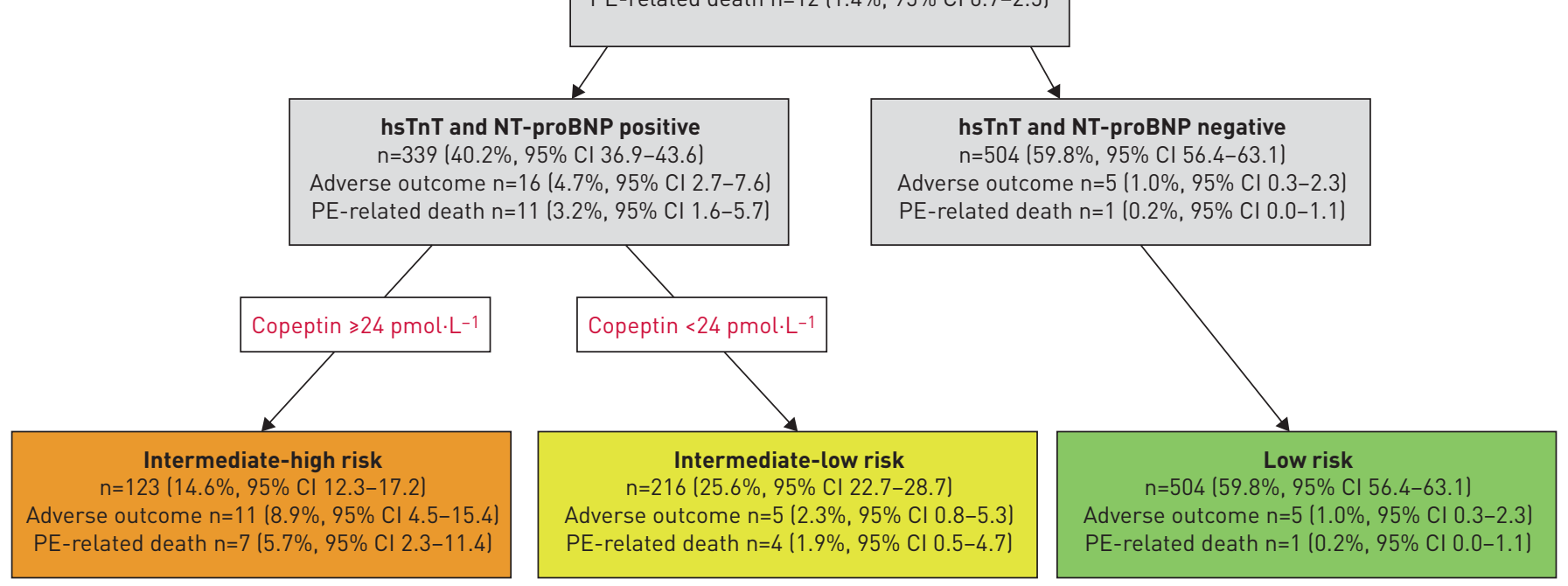

b) 2014 ESC algorithm

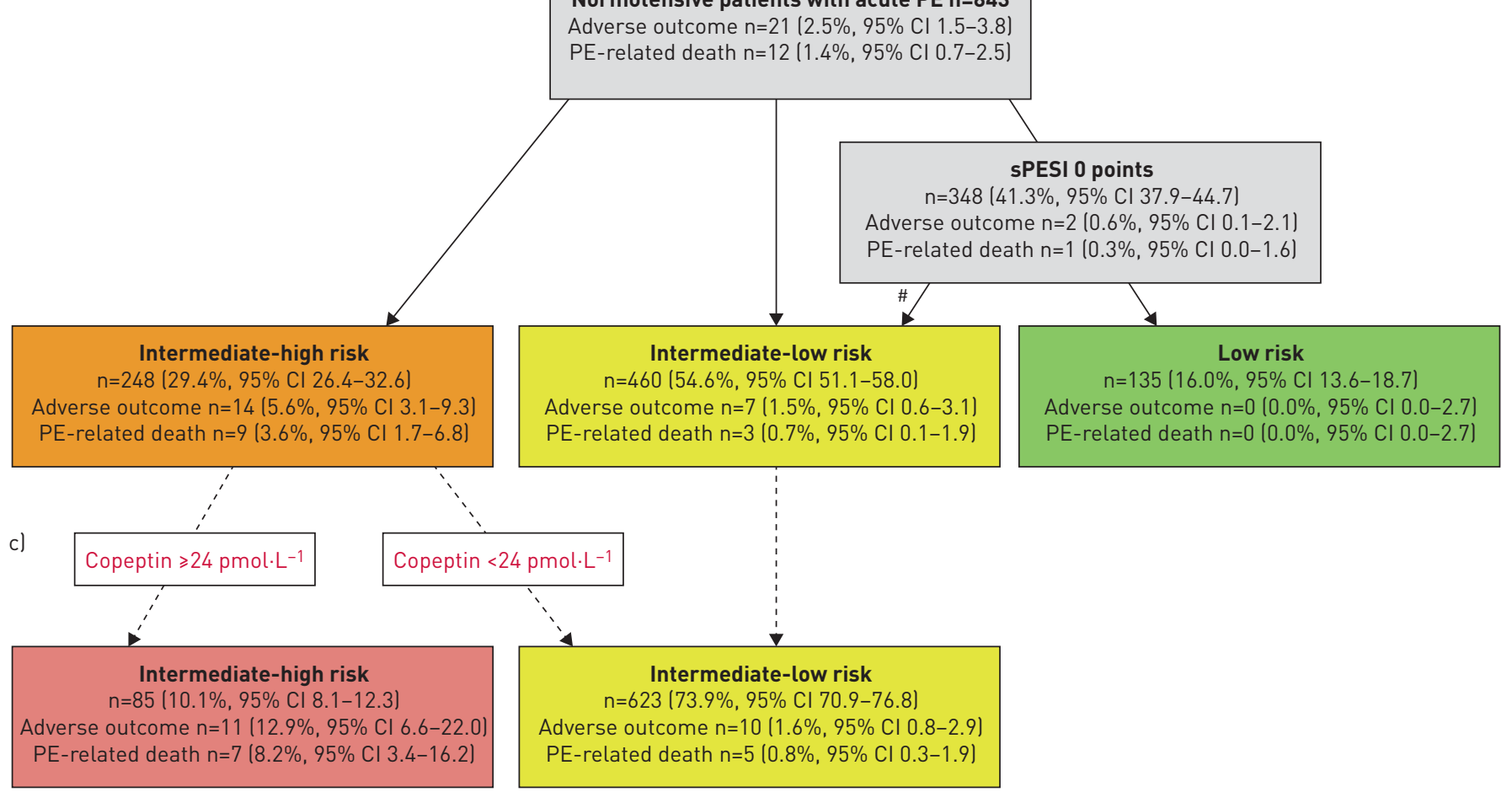

FIGURE 2 Risk assessment strategies for normotensive pulmonary embolism (PE) patients. a) Risk assessment using a biomarker-based strategy based on high-sensitivity troponin T (hsTnT), N-terminal pro-brain natriuretic peptide (NT-proBNP) and copeptin. b) Risk assessment as proposed by the 2014 European Society of Cardiology (ESC) guideline. c) Adding measurement of copeptin in patients classified as intermediate-high risk by the 2014 ESC algorithm helps to identify patients with an increased risk for an adverse outcome. *: according to the 2014 ESC algorithm, patients with a simplified Pulmonary Embolism Severity Index (SPESI) of 0 points and elevated hsTnT or NT-proBNP plasma concentrations were reclassified as intermediate-low risk. A "positive" hsTnT and NT-proBNP test refers to plasma concentrations $\geqslant 14 \mathrm{pg} \cdot \mathrm{mL}^{-1}$ and $\geqslant 600 \mathrm{pg} \cdot \mathrm{mL}^{-1}$, respectively.

had the highest specificity among all tested variables, which may enable copeptin to identify normotensive $\mathrm{PE}$ patients at higher risk. However, as indicated by the moderate individual prognostic performance (figure 1 and table 2), none of the biomarkers should be used alone to risk stratify patients for guidance of therapeutic management. 

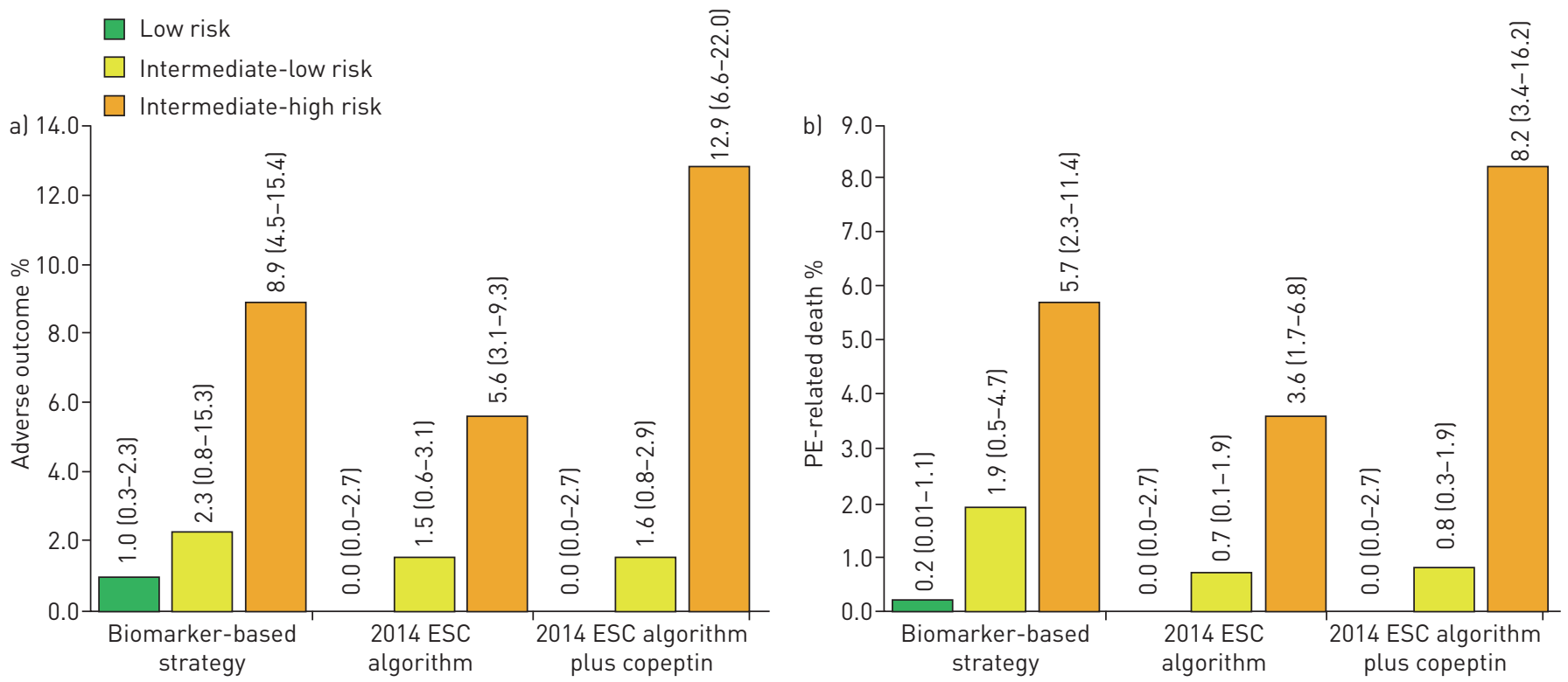

FIGURE 3 Percentage of patients with a) an adverse outcome and b) pulmonary embolism (PE)-related death in each risk category using different risk assessment strategies. All tested strategies were able to stratify patients according to their risk of an adverse outcome and PE-related death. Adding copeptin measurement in intermediate-high-risk patients to the 2014 European Society of Cardiology (ESC) algorithm identified a subgroup of patients with the highest rate of an adverse outcome and PE-related death.

\section{A novel stepwise biomarker-based strategy}

Due to the complex and time-, labour- and potentially cost-intensive approach of the algorithm proposed by the 2014 ESC guideline (requiring three steps with calculation of the sPESI, laboratory testing and imaging procedures), we have developed a novel and simple stepwise biomarker-based strategy [9]. In the derivation study, using the biomarker-based approach instead of the 2014 ESC classification [9] classified more patients as low risk, none of whom reached the primary outcome. Additionally, more patients in the intermediate-high-risk group had an adverse outcome [9]. The present multicentre study allows these findings to be validated. The novel stepwise biomarker-based strategy identified a small group of patients at intermediate-high risk (14.6\%) with a rate of $8.6 \%(95 \%$ CI $4.5-15.4)$ of PE-related death or complications (OR 7.0, 95\% CI 2.9-16.8, p<0.001; figure 2a). Although this proportion is lower than the rate of $20 \%$ of adverse outcomes reported in the derivation study [9], the intermediate-high-risk group still had a higher rate of adverse outcomes when classified by the stepwise biomarker-based strategy rather than by the 2014 ESC algorithm (5.6\%, 95\% CI 3.1-9.3; OR 5.0, 95\% CI 2.0-12.6; p=0.001) or the Bova score $(5.4 \%$, 95\% CI 1.5-13.3; OR 2.5, 95\% CI 0.8-7.7; $\mathrm{p}=0.104)$. On the other end of the risk spectrum, as many as $59.8 \%$ of patients were classified in the low-risk group using the biomarker-based strategy with an acceptable rate of an adverse outcome $(1.0 \%, 95 \%$ CI $0.3-2.3)$ while only $16 \%$ of patients were classified in the low-risk group by the 2014 ESC algorithm; none (95\% CI 0.0-2.7) of these patients had an adverse outcome. Of note, because hsTnT and NT-proBNP were measured in all patients, the number of patients reclassified from low risk to intermediate-low risk might have been higher than in real-world scenarios [19]. Although routine performance of imaging or laboratory testing in the presence of a sPESI of 0 is not considered necessary by current ESC guidelines [1], evidence is accumulating that especially younger PE patients with fewer comorbidities might be misclassified as low risk if further assessment of RV (dys)function is withheld [20]. Thus, risk stratification of PE patients for guidance of the therapeutic strategy should be based on an assessment of the disease-specific prognosis (e.g. PE-related death or complications) if aiming to identify candidates for thrombolytic therapy, and on an assessment of overall prognosis (e.g. by the use of the sPESI [2] or the RIETE score [21]) if aiming to identify candidates for home treatment. Of course, before final therapeutic decisions can be made, the respective treatment-related risks (e.g. risk of bleeding when considering thrombolysis or choosing the optimal anticoagulant strategy and risks related to ambulatory care if considering early discharge) should be assessed.

Improving risk stratification based on the 2014 ESC algorithm by adding copeptin

The Pulmonary Embolism Thrombolysis (PEITHO) study [22] demonstrated that the combined primary endpoint of death or haemodynamic decompensation within 7 days can be reduced by $46 \%$ by administrating a fibrinolytic therapy to normotensive PE patients with RV dysfunction and troponin 
elevation. However, this benefit was achieved at the cost of increased intracranial and major extracranial bleeding [22]. Consequently, the 2014 ESC guideline does not recommend initial thrombolysis in haemodynamically stable PE patients, but does recommend close monitoring of patients in the intermediate-high-risk group to permit early detection of signs of haemodynamic compromise to initiate timely (rescue) thrombolysis [1]. While this recommendation appears to be a reasonable consequence of the results from the PEITHO study and findings from further studies (summarised in [23]), one may be puzzled by the comparably low rate of death and haemodynamic decompensation of $5.6 \%$ in the placebo arm of PEITHO. In comparison, recent derivation cohort studies developing combination models for the identification of intermediate-high-risk PE patients reported complication rates of $>20 \%$ in the high-risk groups (PREP score, 22.2\% [24]; FAST score, 20.5\% [25]; Bova score, 29.2\% [3]). If we assume the same relative risk reduction in these "truly" intermediate-high-risk patients, the risk-to-benefit ratio could be tipped in favour of thrombolytic therapy [26]. Thus, in the present study, we used copeptin $\geqslant 24 \mathrm{pmol} \cdot \mathrm{L}^{-1}$ to further identify patients at intermediate-high risk classified by the 2014 ESC algorithm at highest risk (figure 2c). A small group of higher risk patients (10.1\% of the overall cohort) with a rate of adverse outcome of $12.9 \%$ (95\% CI 6.6-22.0) and of PE-related death of $8.2 \%$ (95\% CI 3.4-16.2) was identified. However, whether intermediate-high-risk patients identified based on copeptin or other combination models will benefit from more aggressive treatment remains to be tested and demonstrated in appropriately designed trials. Of note, and as shown and discussed in the supplementary material and table 3, copeptin can also be used instead of imaging modalities in the 2014 ESC algorithm for risk stratification.

Some limitations deserve consideration: first, the rate of patients with PE-related death (1.4\%, 95\% CI 0.7-2.5) or PE-related complications $(2.5 \%, 95 \%$ CI $1.5-3.8)$ was lower than in other cohort studies $[3,5]$ and the derivation studies $[9,17]$, and below the suggested number of events for external validation of a prognostic model $[27,28]$. However, statistical analyses revealed satisfactory results given the large overall patient number and we were able to confirm the prognostic impact of copeptin, alone or integrated in risk assessment strategies, as well as findings from the two derivation studies [9, 17]. Additionally, a pooled analysis of the derivation and the validation cohort with 36 adverse outcomes $(3.2 \%, 95 \%$ CI $2.3-4.5)$ in 1111 patients provided comparable results (shown in the supplementary material). Second, due to the post hoc study design and inclusion of patients from 12 sites in three countries, echocardiographic criteria for the definition of RV dysfunction differed (as explained in detail in the supplementary material). But given the lack of an accepted standardised definition of RV dysfunction on TTE in general, this does more likely reflect current practice under real-world conditions rather than a limitation. Finally, acknowledging that the implementation of copeptin in clinical routine may constitute a logistical challenge for laboratories because its measurement requires a separate analyser presumably not available in most (smaller) hospitals, further studies should address the cost-effectiveness of copeptin measurements.

In conclusion, copeptin may improve risk stratification of normotensive PE patients if integrated in a simple stepwise biomarker-based risk assessment strategy (combining different information obtained from hsTnT, NT-proBNP and copeptin) and if measured on top of the 2014 ESC algorithm. Pending confirmation by an adequately designed management trial, copeptin appears especially useful for the identification of normotensive PE patients with a higher risk of PE-related adverse outcomes who might be candidates for more aggressive treatment strategies with a risk-to-benefit ratio in favour of a thrombolytic therapy.

Acknowledgements: We thank Dr Irene Schmidtmann (Institute for Medical Biostatistics, Epidemiology and Informatics (IMBEI), University Medical Centre Mainz, Johannes Gutenberg-University Mainz, Germany) for expert statistical advice.

Support statement: BRAHMS GmbH, part of Thermo Fisher Scientific, Hennigsdorf/Berlin, Germany provided all necessary reagents for the measurement of copeptin plasma concentrations and financial support for other biomarker testing. The sponsor was neither involved in biomarker measurements, statistical analyses, writing of the manuscript nor had any influence on the scientific contents of this publication. This study was supported by the German Federal Ministry of Education and Research (BMBF 01EO1503). The authors are responsible for the contents of this publication. Funding information for this article has been deposited with the Crossref Funder Registry.

Conflict of interest: K. Hellenkamp reports grants from BRAHMS GmbH, part of Thermo Fisher Scientific, Hennigsdorf/Berlin, Germany, during the conduct of the study. P. Pruszczyk has nothing to disclose. D. Jiménez has nothing to disclose. A. Wyzgal has nothing to disclose. D. Barrios has nothing to disclose. M. Ciurzynski has nothing to disclose. R. Morillo has nothing to disclose. L. Hobohm reports grants from the German Federal Ministry of Education and Research (BMBF 01EO1503), during the conduct of the study. K. Keller reports grants from the German Federal Ministry of Education and Research (BMBF 01EO1503), during the conduct of the study. K. Kurnicka has nothing to disclose. M. Kostrubiec has nothing to disclose. R. Wachter reports grants from BRAHMS GmbH, part of Thermo Fisher Scientific, Hennigsdorf/Berlin, Germany, during the conduct of the study; personal fees and other from CVRx; grants, personal fees and other from Boehringer Ingelheim; personal fees and other from Bayer; personal fees and other from Servier; personal fees and other from Novartis; other from Johnson and Johnson; grants from European Union; 
grants from Bundesministerium für Bildung und Forschung; personal fees from Pfizer; and personal fees and other from Medtronic, outside the submitted work. G. Hasenfuß reports grants from BRAHMS GmbH, part of Thermo Fisher Scientific, Hennigsdorf/Berlin, Germany, during the conduct of the study; other from Servier, other from Impulse Dynamics, other from Novartis, other from AstraZeneca, other from Corvia, and other from Vifor Pharma, outside the submitted work. S. Konstantinides reports grants and personal fees from Bayer HealthCare, grants and personal fees from Boehringer Ingelheim, grants and personal fees from Actelion, grants from Daiichi Sankyo, grants and personal fees from Pfizer-Bristol-Myers Squibb, and personal fees from MSD, outside the submitted work. M. Lankeit reports grants from German Federal Ministry of Education and Research (BMBF 01EO1503) and grants from BRAHMS GmbH, part of Thermo Fisher Scientific, Hennigsdorf/Berlin, Germany, during the conduct of the study; personal fees from Actelion, personal fees from Bayer, personal fees from Daiichi Sankyo, personal fees from MSD, and personal fees from Pfizer-Bristol-Myers Squibb, outside the submitted work.

\section{References}

1 Konstantinides SV, Torbicki A, Agnelli G, et al. 2014 ESC guidelines on the diagnosis and management of acute pulmonary embolism. Eur Heart J 2014; 35: 3033-3069.

2 Jiménez D, Aujesky D, Moores L, et al. Simplification of the pulmonary embolism severity index for prognostication in patients with acute symptomatic pulmonary embolism. Arch Intern Med 2010; 170: 1383-1389.

3 Bova C, Sanchez O, Prandoni P, et al. Identification of intermediate-risk patients with acute symptomatic pulmonary embolism. Eur Respir J 2014; 44: 694-703.

4 Dellas $\mathrm{C}$, Tschepe $\mathrm{M}$, Seeber V, et al. A novel H-FABP assay and a fast prognostic score for risk assessment of normotensive pulmonary embolism. Thromb Haemost 2014; 111: 996-1003.

5 Hobohm L, Hellenkamp K, Hasenfuß G, et al. Comparison of risk assessment strategies for not-high-risk pulmonary embolism. Eur Respir J 2016; 47: 1170-1178.

6 Jiménez D, Lobo JL, Fernandez-Golfin C, et al. Effectiveness of prognosticating pulmonary embolism using the ESC algorithm and the Bova score. Thromb Haemost 2016; 115: 827-834.

7 Lankeit $M$, Jiménez $\mathrm{D}$, Kostrubiec $\mathrm{M}$, et al. Predictive value of the high-sensitivity troponin $\mathrm{T}$ assay and the simplified Pulmonary Embolism Severity Index in haemodynamically stable patients with acute pulmonary embolism: a prospective validation study. Circulation 2011; 124: 2716-2724.

8 Lankeit M, Jiménez D, Kostrubiec $\mathrm{M}$, et al. Validation of $\mathrm{N}$-terminal pro-brain natriuretic peptide cut-off values for risk stratification of pulmonary embolism. Eur Respir J 2014; 43: 1669-1677.

9 Hellenkamp K, Schwung J, Rossmann H, et al. Risk stratification of normotensive pulmonary embolism: prognostic impact of copeptin. Eur Respir J 2015; 46: 1701-1710.

10 Bolignano D, Cabassi A, Fiaccadori E, et al. Copeptin (CTproAVP), a new tool for understanding the role of vasopressin in pathophysiology. Clin Chem Lab Med 2014; 52: 1447-1456.

11 Reichlin T, Hochholzer W, Stelzig C, et al. Incremental value of copeptin for rapid rule out of acute myocardial infarction. J Am Coll Cardiol 2009; 54: 60-68.

12 Maisel A, Mueller C, Neath SX, et al. Copeptin helps in the early detection of patients with acute myocardial infarction: primary results of the CHOPIN trial (Copeptin Helps in the early detection Of Patients with acute myocardial INfarction). J Am Coll Cardiol 2013; 62: 150-160.

13 Khan SQ, Dhillon OS, O'Brien RJ, et al. C-terminal provasopressin (copeptin) as a novel and prognostic marker in acute myocardial infarction: Leicester Acute Myocardial Infarction Peptide (LAMP) study. Circulation 2007; 115: 2103-2110.

14 Voors AA, von Haehling S, Anker SD, et al. C-terminal provasopressin (copeptin) is a strong prognostic marker in patients with heart failure after an acute myocardial infarction: results from the OPTIMAAL study. Eur Heart $J$ 2009; 30: 1187-1194.

15 Neuhold S, Huelsmann M, Strunk G, et al. Comparison of copeptin, B-type natriuretic peptide, and amino-terminal pro-B-type natriuretic peptide in patients with chronic heart failure: prediction of death at different stages of the disease. J Am Coll Cardiol 2008; 52: 266-272.

16 Nickel NP, Lichtinghagen R, Golpon H, et al. Circulating levels of copeptin predict outcome in patients with pulmonary arterial hypertension. Respir Res 2013; 14: 130.

17 Wyzgał A, Koć M, Pacho S, et al. Plasma copeptin for short term risk stratification in acute pulmonary embolism. J Thromb Thrombolysis 2016; 41: 563-568.

18 Vuilleumier N, Simona A, Méan M, et al. Comparison of cardiac and non-cardiac biomarkers for risk stratification in elderly patients with non-massive pulmonary embolism. PLoS One 2016; 11: e0155973.

19 Becattini C, Agnelli G, Lankeit M, et al. Acute pulmonary embolism: mortality prediction by the 2014 European Society of Cardiology risk stratification model. Eur Respir J 2016; 48: 780-786.

20 Hellenkamp K, Kaeberich A, Schwung J, et al. Risk stratification of normotensive pulmonary embolism based on the sPESI - does it work for all patients? Int J Cardiol 2015; 197: 162-163.

21 Maestre A, Trujillo-Santos J, Riera-Mestre A, et al. Identification of low-risk patients with acute symptomatic pulmonary embolism for outpatient therapy. Ann Am Thorac Soc 2015; 12: 1122-1129.

22 Meyer G, Vicaut E, Danays T, et al. Fibrinolysis for patients with intermediate-risk pulmonary embolism. $N$ Engl J Med 2014; 370: 1402-1411.

23 Marti C, John G, Konstantinides S, et al. Systemic thrombolytic therapy for acute pulmonary embolism: a systematic review and meta-analysis. Eur Heart J 2015; 36: 605-614.

24 Sanchez O, Trinquart L, Caille V, et al. Prognostic factors for pulmonary embolism: the prep study, a prospective multicentre cohort study. Am J Respir Crit Care Med 2010; 181: 168-173.

25 Lankeit M, Friesen D, Schäfer K, et al. A simple score for rapid risk assessment of non-high-risk pulmonary embolism. Clin Res Cardiol 2013; 102: 73-80.

26 Meyer G, Planquette B, Sanchez O. Risk stratification of pulmonary embolism: clinical evaluation, biomarkers or both? Eur Respir J 2015; 46: 1551-1553.

27 Vergouwe Y, Steyerberg EW, Eijkemans MJ, et al. Substantial effective sample sizes were required for external validation studies of predictive logistic regression models. J Clin Epidemiol 2005; 58: 475-483.

28 Collins GS, Ogundimu EO, Altman DG. Sample size considerations for the external validation of a multivariable prognostic model: a resampling study. Stat Med 2016; 35: 214-226. 